images when DCE-MRI was included. This technique could be used by radiologists who have less experience with MRI of the prostate. Studies including larger numbers of radiologists will help to show whether these results can be applied generally.

\section{Caroline Barranco}

Original article Fütterer JJ et al. (2005) Staging prostate cancer with dynamic contrast-enhanced endorectal MR imaging prior to radical prostatectomy: experienced versus

less experienced readers. Radiology 237: 541-549

\section{Sacral neuromodulation: factors that affect cure rates for urge incontinence}

Hints from previous studies that sacral neuromodulation might be less effective as a treatment for intractable urinary urge incontinence in older individuals have been confirmed in a prospective US study. Amundsen et al. aimed to determine which health and clinical variables are associated with lower rates of cure for this condition.

Cure was defined as no daily leakage episodes following implantation of a permanent electrode. The study cohort comprised 55 patients (age range 29-83 years) with urinary urge incontinence that was refractory to conservative and pharmacologic management, who had responded favorably to a successful test stimulation. Of these 55 patients, only four were male.

The cure rate was markedly lower for older individuals: $65 \%$ of those aged $\leq 55$ were cured, compared with $37 \%$ of those older than $55(P<0.05)$. A similar trend was shown in the subgroup of patients with a neurologic condition. Over the whole cohort, both age groups were similar in terms of preimplantation severity of incontinence and comorbidities; however, the presence of three or more chronic conditions was associated with lower rates of cure in both older and younger individuals.

The authors speculate that age-related changes in the brain, in the neural control systems for the bladder and in the bladder itself, as observed in human and animal studies, might contribute to the reduced treatment efficacy of sacral neuromodulation in older individuals with urinary urge incontinence. They call for studies evaluating the associations between aging and the neurophysiology of micturition.

Caroline Barranco

Original article Amundsen CL et al. (2005) Sacral neuromodulation for intractable urge incontinence: are there factors associated with cure? Urology 66: 746-750

\section{Kidney paired donation: the answer for patients with incompatible live donors?}

There are an estimated 6,000 patients in the US on the renal transplant waiting list with willing but incompatible donors. Access to transplantation could be improved by implementing a national program of live kidney paired donation (KPD), through which each recipient receives a compatible organ by exchanging with another donor/recipient pair. Successful transplantation outcomes using KPD at Johns Hopkins Hospital highlight the potential benefits of this approach.

A prospective series of paired donations (eight involving two donor/recipient pairs, plus two triple exchanges) enabled 22 patients with donors incompatible for blood type or crossmatch to undergo kidney transplantation. For the five recipients highly sensitized to common human leukocyte antigens as a result of previous transplants, blood transfusions or pregnancies, the probability of receiving a suitable kidney from the deceased donor pool was very low. Nevertheless, only one patient had anti-human-leukocyteantigen antibodies specific to his KPD exchange partner (although at a much lower level than with the originally intended donor) and was given DESENSITIZATION treatment prior to transplantation.

At 6-month follow-up, median serum creatinine was $106.1 \mu \mathrm{mol} / \mathrm{l}(1.2 \mathrm{mg} / \mathrm{dl})$ and, at a median of 13 months following transplantation, $95.5 \%$ of recipients had functioning grafts. Acute cellular rejection developed in four patients, all of whom responded to treatment. No antibodymediated rejection episodes occurred.

Despite logistical and administrative challenges, KPD is successful and cost-efficient. Extending this program to a multicenter donor pool would increase the probability of finding suitable matches.

Rachael Williams

Original article Montgomery RA et al. (2005) Clinical results from transplanting incompatible live kidney donor/recipient pairs using kidney paired donation. JAMA 294: 1655-1663

\section{GLOSSARY} DESENSITIZATION

Enabling incompatible transplants by removing or neutralizing blood group or human-leukocyteantigen-specific antibodies with plasmapheresis and intravenous immunoglobulin 